\title{
Diabetes is associated with genotypically drug-resistant tuberculosis
}

\author{
To the Editor:
}

Diabetes is associated with failure of tuberculosis (TB) treatment, but it is unclear whether this is related to genotypic drug resistance of the infecting mycobacteria. We used whole genome sequencing (WGS) to examine 1365 known drug resistance mutations in 896 Mycobacterium tuberculosis isolates from TB patients that were screened for diabetes using HbAlc testing as part of the TANDEM project [1]. Ethical approval was received from the London School of Hygiene and Tropical Medicine and institutional review boards in Indonesia and Peru. In Peru we selected all available M. tuberculosis isolates from TANDEM patients (44 with and 445 without diabetes), and in Indonesia we selected all available isolates from diabetic patients $(n=115)$ plus a subset of isolates from non-diabetic patients $(n=292)$ from the same clinics, during the same time period, frequency-matched by age. We used TB Profiler version 0.3.8 [2] to determine $M$. tuberculosis lineage and drug resistance. A phylogeny was constructed using PhyML version 3.0 [3], and the minimum pairwise distance for isolates was calculated separately for patients with and without diabetes, stratified by country. We examined if diabetes was associated with genotypic drug resistance against individual drugs or with multidrug-resistant (MDR)-TB for the two countries separately and combined, with multilevel multivariable logistic regression, taking into account the country of origin and adjusting for age, gender, HIV infection, previous TB treatment and M. tuberculosis lineage. In additional analyses we examined the effect of HbAlc level, and stratified results for new versus known diabetes. For comparison of specific drug resistance mutations, we used univariate and country-stratified analysis because of small numbers. We also addressed the hypothesis that isolates from patients with diabetes would have fewer mutations compensating for loss of fitness associated with drug resistance, noting that diabetes leads to lower host immune defence against $M$. tuberculosis [4]. For this analysis, all non-synonymous single nucleotide polymorphisms (SNPs) in $r p o A, r p o B$ outside the rifampicin resistance-determining region (RRDR), $r p o C$, $a h p C$ promoter region and $u b i A$ were considered as mutations potentially compensating for the loss of fitness caused by drug resistance mutations [5]. More detailed methods are provided as supplementary material (https://doi.org/10.6084/m9.figshare.9884303.v2).

Diabetes was more common among Indonesian patients, and drug resistance more common in Peru. TB patients with diabetes were older, more often female, and slightly heavier than TB patients without diabetes, and fewer diabetic patients reported a previous history of TB treatment compared to those without diabetes (table 1). Diabetes was not associated with a particular M. tuberculosis lineage or with genotypic clustering; the median minimum pairwise distance for isolates from diabetic and non-diabetic patients was 164 and 161 SNPs in Indonesia, and 98 and 67 SNPs in Peru, respectively. Drug resistance mutations were found in isolates of 21/115 (18\%) diabetic and 44/292 (15\%) non-diabetic patients in Indonesia, and 17/44 (39\%) diabetic and 88/445 (20\%) non-diabetic patients in Peru. In multilevel multivariable logistic regression, diabetes was the only factor significantly associated with genotypic drug resistance against at least one drug (OR 1.8, 95\% CI 1.1-2.9). The association between diabetes and drug resistance was similar for patients with new (adjusted OR 2.0, 95\% CI 0.9-4.4) and previously diagnosed diabetes (OR 1.7, 95\% CI 0.98-2.9), and not dependent on the HbA1c level (not shown). The relationship between diabetes and resistance was still present after exclusion of 19 HIV-infected patients (adjusted OR $1.8,95 \%$ CI 1.1-2.9). At the level of individual drugs, diabetes was significantly associated with rifampicin resistance (OR 2.5, 95\% CI 1.2-5.3) (supplementary figure, https://doi.org/10.6084/m9.figshare.9884303.v2), and also among patients not previously treated for TB (OR 3.1, 95\% CI 1.2-8.3; data not shown). We also found more fluoroquinolone resistance in diabetic TB patients, and this difference reached statistical

@ERSpublications

In a study in Peru and Indonesia, diabetes was associated with an increased risk of tuberculosis caused by Mycobacterium tuberculosis strains with resistance mutations, particularly against rifampicin, and this was not explained by previous TB treatment http://bit.ly/2QCvUEM

Cite this article as: Ruesen C, Chaidir L, Ugarte-Gil C, et al. Diabetes is associated with genotypically drug-resistant tuberculosis. Eur Respir J 2020; 55: 1901891 [https://doi.org/10.1183/13993003.01891-2019]. 
TABLE 1 Patient characteristics and drug resistance mutations stratified for country and diabetes

\begin{tabular}{|c|c|c|c|c|}
\hline & \multicolumn{2}{|c|}{ Indonesia } & \multicolumn{2}{|c|}{ Peru } \\
\hline & Diabetes & No diabetes & Diabetes & No diabetes \\
\hline Subjects n & 115 & 292 & 44 & 445 \\
\hline Male gender & $52(45 \%)$ & $168(58 \%)$ & $25(57 \%)$ & $268(60 \%)$ \\
\hline Age years & $50(45-58)$ & $39(33-48)$ & $52(42-59)$ & $28(22-39)$ \\
\hline Previous TB treatment & $24(21 \%)$ & $79(27 \%)$ & $6(14 \%)$ & $96(22 \%)$ \\
\hline Previously diagnosed diabetes & $83(72.2 \%)$ & NA & $32(72.7 \%)$ & NA \\
\hline Newly diagnosed diabetes & $32(27.8 \%)$ & NA & $12(27.3 \%)$ & NA \\
\hline HIV infection & 0 & 0 & $1(2.3 \%)$ & $18(4.0 \%)$ \\
\hline \multicolumn{5}{|l|}{ M. tuberculosis lineage } \\
\hline East-Asian & $40(34.8 \%)$ & $90(30.8 \%)$ & $7(15.9 \%)$ & $59(13.3 \%)$ \\
\hline Euro-American & $69(60.0 \%)$ & $178(61.0 \%)$ & $37(84.1 \%)$ & $386(86.7 \%)$ \\
\hline Indo-Oceanic & $5(4.3 \%)$ & $24(28.2 \%)$ & 0 & 0 \\
\hline M. bovis & $1(0.9 \%)$ & 0 & 0 & 0 \\
\hline \multicolumn{5}{|l|}{ Isoniazid resistance } \\
\hline katG & $8(7.0 \%)$ & $19(6.5 \%)$ & $6(13.6 \%)$ & $48(10.8 \%)$ \\
\hline Rv1482c-fabG1 & $3(2.6 \%)$ & $10(3.4 \%)$ & $8(18.2 \%)^{* *}$ & $23(5.2 \%)^{* *}$ \\
\hline$a h p C$ & 0 & 0 & $1(2.3 \%)$ & 0 \\
\hline \multicolumn{5}{|l|}{ Rifampicin resistance } \\
\hline rpoB & $9(7.8 \%)$ & $10(3.4 \%)$ & $7(15.9 \%)$ & $42(9.4 \%)$ \\
\hline \multicolumn{5}{|l|}{ Ethambutol resistance } \\
\hline embB & $3(2.6 \%)$ & $6(2.1 \%)$ & $5(11.4 \%)$ & $30(6.7 \%)$ \\
\hline embC-embA & 0 & 0 & $1(2.3 \%)$ & $6(1.3 \%)$ \\
\hline$e m b R$ & 0 & $1(0.3 \%)$ & 0 & 0 \\
\hline \multicolumn{5}{|l|}{ Streptomycin resistance } \\
\hline$r p s L$ & $4(3.5 \%)$ & $5(1.7 \%)$ & $1(2.3 \%)$ & $16(3.6 \%)$ \\
\hline rrs & $1(0.9 \%)$ & $2(0.7 \%)$ & 0 & $5(1.1 \%)$ \\
\hline \multicolumn{5}{|l|}{ Pyrazinamide resistance } \\
\hline pncA & $7(6.1 \%)$ & $14(4.8 \%)$ & $3(6.8 \%)$ & $22(4.9 \%)$ \\
\hline \multicolumn{5}{|l|}{ Ethionamide resistance } \\
\hline Rv1482c-fabG1 & $3(2.6 \%)$ & $10(3.4 \%)$ & $8(18.2 \%)^{* *}$ & $22(4.9 \%)^{* *}$ \\
\hline ethA & 0 & 0 & 0 & $1(0.2 \%)$ \\
\hline \multicolumn{5}{|l|}{ Fluoroquinolone resistance } \\
\hline gyrA & $3(2.6 \%)$ & $3(1.0 \%)$ & $4(9.1 \%)^{*}$ & $7(1.6 \%)^{*}$ \\
\hline gyrB & 0 & 0 & 0 & $2(0.4 \%)$ \\
\hline \multicolumn{5}{|l|}{ Amikacin resistance } \\
\hline rrs & 0 & 0 & 0 & $5(1.1 \%)$ \\
\hline \multicolumn{5}{|l|}{ Capreomycin resistance } \\
\hline tlyA & 0 & $1(0.3 \%)$ & 0 & $7(1.6 \%)$ \\
\hline rrs & 0 & 0 & 0 & $5(1.1 \%)$ \\
\hline \multicolumn{5}{|l|}{ Kanamycin resistance } \\
\hline eis-Rv2417c & $1(0.9 \%)$ & $1(0.3 \%)$ & $1(2.3 \%)$ & $1(0.2 \%)$ \\
\hline rrs & 0 & 0 & 0 & $5(1.1 \%)$ \\
\hline \multicolumn{5}{|c|}{ Para-aminosalicylic acid resistance } \\
\hline folc & 0 & $2(0.7 \%)$ & $1(2.3 \%)$ & $2(0.4 \%)$ \\
\hline thyA & 0 & 0 & 0 & $1(0.2 \%)$ \\
\hline
\end{tabular}

Patient characteristics are presented as $\mathrm{n}(\%)$ or median (interquartile range), unless otherwise stated. Drug resistance data represent $n(\%)$ of isolates with at least one mutation in the respective gene. TB: tuberculosis; NA: not applicable. ${ }^{*}: p<0.05 ;{ }^{* *}$ : $p<0.01$. $p$-values were calculated as Chi-square $p$-values unless the expected number of resistant isolates was $<5$, in which case the Fisher's exact test $p$-value was calculated).

significance for Peru (OR 6.69, 95\% CI 1.37-32.68) (supplementary figure). We did not find evidence of interaction between rifampicin and fluoroquinolone resistance in the multilevel multivariable model ( $\mathrm{p}_{\text {interaction }}=0.232$; data not shown). Finally, although this association did not reach statistical significance, the odds of MDR-TB were twice as high in diabetic versus non-diabetic patients (OR 2.09, 95\% CI $0.92-$ 4.77) (supplementary figure). 
Examining individual drug resistance mutations (table 1), diabetes among TB patients in Peru was associated with more mutations in $R v 1482 c-f a b G 1 \quad(\mathrm{p}<0.01)$, which confers resistance to isoniazid and ethionamide, and $g y r A(\mathrm{p}<0.05)$, which accounts for fluoroquinolone resistance; $r p o B$ mutations leading to rifampicin resistance also appeared more common in diabetes patients $(15.9 \%$ versus $9.4 \% ; \mathrm{p}=0.069)$. In Indonesia, drug resistance was less common, and no significant association was found between diabetes and specific resistance mutations. With regard to compensatory mutations, no differences were found in the association between isoniazid or rifampicin resistance mutations and potential compensatory mutations between patients with and without diabetes, and no interaction was found between diabetes and the presence of compensatory mutations (data not shown).

Our findings are in line with previous studies that used phenotypic drug susceptibility testing (DST) and focused on isoniazid and rifampicin (reviewed by Tegegne et al. [6]). Several factors might account for the observed association between diabetes and drug resistance mutations. First, people with diabetes might be at higher risk of nosocomial transmission of drug-resistant TB in low-resource settings [7]; unfortunately, we did not have the data to compare prior hospitalisation for diabetes compared to non-diabetes $\mathrm{TB}$ patients. Second, lower rifampicin plasma concentrations among diabetes patients that were found in some [8] but not all [9] studies might lead to acquisition of drug resistance. However, all isolates in our study were collected before start of treatment, only $26 \%$ of patients with a drug-resistant isolate reported an episode of previous TB treatment that might have resulted in acquired drug resistance mutations, and differences were also present among patients with a first episode of TB. We cannot exclude misclassification of TB treatment history, as this was self-reported and as some patients previously treated for TB may in fact have had another illness. However, it is unlikely that possible misclassification of patients regarding TB treatment history explains the association between drug resistance mutations and diabetes, as it was evident both among patients with and without a history of previous TB treatment. Third, recent papers have found an interaction between drug resistance and cellular immunometabolism [10], and this interaction might be altered by diabetes. Fourth, similar to HIV, reduced host defence in people with diabetes might increase the risk of developing active TB caused by $M$. tuberculosis strains with drug resistance mutations associated with loss of fitness [11], although we could not confirm this when looking at previously reported resistance-compensating mutations. The trend towards more fluoroquinolone resistance in $\mathrm{TB}$ patients with diabetes could be related to frequent use of fluoroquinolones for respiratory infections, which may be more common for those with diabetes [12].

In contrast to studies using phenotypic DST, WGS allowed us not only to investigate the association between diabetes and drug resistance at the gene level, but also to take the diverse $M$. tuberculosis genetic background into account. However, our study could not prove if diabetes is associated with more transmission of drug resistance, as the sampling fraction of $M$. tuberculosis isolates was probably too low to identify transmission clusters. Besides higher rates of transmission, diabetes may also lead to more $\mathrm{TB}$ reactivation caused by drug-resistant strains.

In summary, for the first time, we used M. tuberculosis WGS data from two countries to study the association between diabetes and genotypic drug resistance in TB patients. Diabetes was associated with an increased risk of disease caused by strains with resistance mutations, particularly against rifampicin, but also against isoniazid, ethionamide and fluoroquinolones. Higher rates of resistance mutations among diabetic TB patients could not be explained by previous TB treatment; the association between diabetes and resistance was also evident among patients with a first episode of TB. TB patients with diabetes should be prioritised for DST in settings where it is not performed for all patients, and more (molecular) epidemiological and mechanistic studies are needed to unravel the factors explaining the association between diabetes and TB drug resistance.

Carolien Ruesen $\odot^{1}$, Lidya Chaidir ${ }^{2,3}$, Cesar Ugarte-Gil ${ }^{4,5,6}$, Jakko van Ingen $\odot^{1}$, Julia A. Critchley ${ }^{7}$, Philip C. Hill ${ }^{8}$, Rovina Ruslami', Prayudi Santoso ${ }^{9}$, Martijn A. Huynen ${ }^{10}$, Hazel M. Dockrell $\oplus^{11}$, David A.J. Moore ${ }^{6,12}$, Bachti Alisjahbana ${ }^{3,9}$ and Reinout van Crevel $^{1,13}$

${ }^{1}$ Radboudumc Center for Infectious Diseases, Radboud Institute for Health Sciences, Radboudumc, Nijmegen, The Netherlands. ${ }^{2}$ Dept of Biomedical Science, Faculty of Medicine, Universitas Padjadjaran, Bandung, Indonesia. ${ }^{3}$ Infectious Disease Research Center, Faculty of Medicine, Universitas Padjadjaran, Bandung, Indonesia. ${ }^{4}$ Facultad de Medicina, Universidad Peruana Cayetano Heredia, Lima, Peru. ${ }^{5}$ Instituto de Medicina Tropical Alexander von Humboldt, Universidad Peruana Cayetano Heredia, Lima, Peru. ${ }^{6} \mathrm{~TB}$ Centre, London School of Hygiene and Tropical Medicine, London, UK. ${ }^{7}$ Population Health Research Institute, St. George's, University of London, London, UK. ${ }^{8}$ Centre for International Health, Dunedin School of Medicine, University of Otago, Dunedin, New Zealand. ${ }^{9}$ Dept of Internal Medicine, Faculty of Medicine, Universitas Padjadjaran/Hasan Sadikin Hospital, Bandung, Indonesia. ${ }^{10}$ Centre for Molecular and Biomolecular Informatics, Radboud Institute for Molecular Life Sciences, Radboudumc, Nijmegen, The Netherlands. ${ }^{11}$ Dept of Infection Biology and TB Centre, London School of Hygiene and Tropical Medicine, London, UK. ${ }^{12}$ Universidad Peruana Cayetano Heredia, Lima, Peru. ${ }^{13}$ Centre for Tropical Medicine and Global Health, Nuffield Dept of Medicine, University of Oxford, Oxford, UK. 
Correspondence: Reinout van Crevel, Dept of Internal Medicine, Radboudumc, Nijmegen, The Netherlands. PO Box 9101, 6500 HB Nijmegen, The Netherlands. E-mail: Reinout.vancrevel@radboudumc.nl

Received: 30 July 2019 | Accepted after revision: 16 Nov 2019

Acknowledgements: We thank the Oxford Genomics Centre at the Welcome Centre for Human Genetics (funded by Welcome Trust grant reference $203141 / \mathrm{Z} / 16 / \mathrm{Z}$ ) for the generation and initial processing of the whole genome sequencing data.

Support statement: This work was supported by the TANDEM project, which was funded by the European Union's Seventh Framework Programme (FP7/2007-2013) under grant agreement number 305279. Whole genome sequencing: CRyPTIC consortium, funded by a Welcome Trust/Newton Fund-MRC Collaborative Award (200205/Z/15/Z) and the Bill and Melinda Gates Foundation Trust (OPP1133541).

Conflict of interest: None declared.

\section{References}

1 Ugarte-Gil C, Alisjahbana B, Ronacher K, et al. Diabetes mellitus among pulmonary tuberculosis patients from four TB-endemic countries: the TANDEM study. Clin Infect Dis 2019; 70: 780-788.

2 Coll F, Preston M, Guerra-Assuncao JA, et al. PolyTB: a genomic variation map for Mycobacterium tuberculosis. Tuberculosis (Edinb) 2014; 94: 346-354.

3 Guindon S, Dufayard JF, Lefort V, et al. New algorithms and methods to estimate maximum-likelihood phylogenies: assessing the performance of PhyML 3.0. Syst Biol 2010; 59: 307-321.

4 Ronacher K, van Crevel R, Critchley JA, et al. Defining a research agenda to address the converging epidemics of tuberculosis and diabetes. Chest 2017; 152: 174-180.

5 Merker M, Barbier M, Cox $\mathrm{H}$, et al. Compensatory evolution drives multidrug-resistant tuberculosis in Central Asia. Elife 2018; 7: e38200.

6 Tegegne BS, Mengesha MM, Teferra AA, et al. Association between diabetes mellitus and multi-drug-resistant tuberculosis: evidence from a systematic review and meta-analysis. Syst Rev 2018; 7: 161.

7 van Crevel R, van de Vijver S, Moore DAJ. The global diabetes epidemic: what does it mean for infectious diseases in tropical countries? Lancet Diabetes Endocrinol 2017; 5: 457-468.

8 Alkabab Y, Keller S, Dodge D, et al. Early interventions for diabetes related tuberculosis associate with hastened sputum microbiological clearance in Virginia, USA. BMC Infect Dis 2017; 17: 125.

9 Ruslami R, Nijland HM, Adhiarta IG, et al. Pharmacokinetics of antituberculosis drugs in pulmonary tuberculosis patients with type 2 diabetes. Antimicrob Agents Chemother 2010; 54: 1068-1074.

10 Howard NC, Marin ND, Ahmed M, et al. Mycobacterium tuberculosis carrying a rifampicin drug resistance mutation reprograms macrophage metabolism through cell wall lipid changes. Nat Microbiol 2018; 3: 1099-1108.

11 Dheda K, Gumbo T, Maartens G, et al. The epidemiology, pathogenesis, transmission, diagnosis, and management of multidrug-resistant, extensively drug-resistant, and incurable tuberculosis. Lancet Respir Med 2017; 5: 291-360.

12 Long $\mathrm{R}$, Chong $\mathrm{H}$, Hoeppner $\mathrm{V}$, et al. Empirical treatment of community-acquired pneumonia and the development of fluoroquinolone-resistant tuberculosis. Clin Infect Dis 2009; 48: 1354-1360. 